\title{
Rheology, DSC and Volume or Weight Change Induced by Immersion in Solvents for Agarose and Kappa-Carrageenan Gels
}

\author{
Mineo WATASE and Katsuyoshi NiSHINARI* \\ Chemical Research Laboratory, Faculty of Liberal Arts, \\ Shizuoka University, Ohya, Shizuoka 422, Japan \\ *Instrumentation Research Laboratory, National Food Research Institute, \\ Ministry of Agriculture, Forestry and Fisheries, Tsukuba 305, Japan
}

(Received June 25, 1986)

\begin{abstract}
In order to study the gelling ability of kappa-carrageenan and agarose, measurements of dynamic viscoelasticity, differential scanning calorimetry (DSC) and volume or weight change induced by immersion in organic solvents have been made at the range of concentration from 2 to $8 \%(\mathrm{w} / \mathrm{w})$. The results obtained are as follows; (1) The value of dynamic Young's modulus $E^{\prime}$ for agarose gels increases with increasing temperature up to about $35^{\circ} \mathrm{C}$, while it decreases rapidly with increasing temperature above about $35^{\circ} \mathrm{C}$. On the other hand, the value of $E^{\prime}$ for kappa-carrageenan gels decreases with increasing temperature. (2) DSC curves show one endothermic peak which is caused by the melting of gels. The temperature of the endothermic peak accompanying the melting of agarose gels is independent of concentrations, and that of carrageenan gels shifts to higher temperatures with increasing concentrations. (3) The weight or volume change by immersion in dimethyl sulfoxide (DMSO)-water or in ethanol is far more remarkable in kappa-carrageenan gels than in agarose gels. The difference of these kinds of behaviour between agarose and kappa-carrageenan gels is understood mainly by the quantity and position of sulfate side groups in molecules.
\end{abstract}

KEY WORDS Agarose / Kappa-Carrageenan / Dimethyl Sulfoxide /

Differential Scanning Calorimetry / Dynamic Young's Modulus /

Agarose is composed of D-galactose and 3,6-anhydro-L-galactose and little contain electrolytic groups. Kappa-carrageenan is composed of D-galactose, 3,6-anhydro-Dgalactose, and sulfate groups (see Figure 1). Rees and his coworkers ${ }^{1,2}$ proposed the gelation mechanism for kappa-carrageenan as follows: On cooling aqueous kappa-carrageenan solution, a part of the random coil molecules of kappa-carrageenan change into double helix molecules. Then, the aggregation of these double helix molecules forms microcrystalline regions (cross-linking junctions), which are connected by long flexible molecules.

The structure of agarose and kappa-carrageenan is very similar. But, kappa-car-

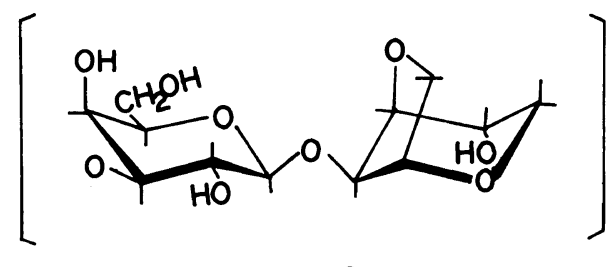

agarose

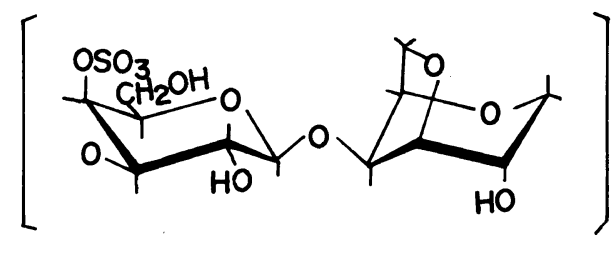

kappa-carrageenan

Figure 1. Molecular structures of agarose and kappacarrageenan. 
rageenan is apt to react with protein, ${ }^{3}$ and the rheological properties are strongly influenced by alkali metal ions, ${ }^{1,2}$ while agarose does not show such a behaviour. ${ }^{4}$ Since kappacarrageenan contains more sulfate groups than agarose, the formation and tight binding of helices and the subsequent aggregation of these helices are hindered in kappacarrageenan because of the electrostatic repulsion of sulfate groups. Therefore, kappacarrageenan gels show the smaller elastic modulus and lower DSC melting temperature as compared with agarose gels. ${ }^{5,6}$

The present authors studied the rheological and thermal properties of agarose and kappa-carrageenan gels containing alkali metal ions ${ }^{4}$ and alkali earth metal ions, ${ }^{7}$ and also the change of viscoelastic coefficients and of volume for these gels by immersion in alkali metal salt solution and alkali earth metal salt solutions. ${ }^{8,9}$ The elastic modulus of kappa-carrageenan gels increased remarkably by addition of these salts or by immersion in these salt solutions. The increase of elastic modulus was more remarkäble in salt solution containing $\mathrm{K}^{+}$or $\mathrm{Cs}^{+}$ions than in solution containing $\mathrm{Li}^{+}$or $\mathrm{Na}^{+}$ions. ${ }^{4}$ The ions of $\mathrm{K}^{+}$or $\mathrm{Cs}^{+}$are considered as structure disordering ions, while ions of $\mathrm{Li}^{+}$or $\mathrm{Na}^{+}$ are considered as structure ordering ions. ${ }^{10}$ Therefore, $\mathrm{K}^{+}$or $\mathrm{Cs}^{+}$shields an electrostatic repulsion of sulfate groups in kappa-carrageenan molecules more directly than $\mathrm{Li}^{+}$ or $\mathrm{Na}^{+}$does, because there would be some intervening water molecules between the sulfate groups and $\mathrm{Li}^{+}$or $\mathrm{Na}^{+}$. Since the electrostatic repulsion of sulfate groups prevents the formation of helices and also the aggregation of helices, the introduction of cations stabilises the kappa-carrageenan gels, and results in an increase of elastic modulus., ${ }^{4}$ Since agarose molecules have no sulfate groups, the rheological properties of agarose gels are hardly influenced by the

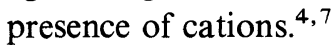

Recently the present authors studied rheo- logical and thermal properties of concentrated gels of agarose $^{5}$ and kappa-carrageenan, ${ }^{6}$ and found the difference in rheological and thermal behaviour of these polymers. The experimental results of dynamic viscoelasticity, the DSC endotherms accompanying gel-sol transition, the change in volume or weight induced by immersion in DMSO$\mathrm{H}_{2} \mathrm{O}$ or ethanol are described in the present paper using agarose and kappa-carrageenan with different origin and preparation from previous studies. ${ }^{5,6}$ Since the gel forming ability of seaweed polysaccharides is strongly influenced not only by the species of seaweeds but also by the season of collecting seaweeds, ${ }^{11}$ the place of collection, ${ }^{12}$ and the method of extraction, ${ }^{13}$ it is necessary to examine the difference of physicochemical properties of agarose and carrageenan gels with different origin and preparation from the previous studies. ${ }^{5,6}$ The study of volume or weight change induced by immersion in organic solvents will clarify the relation between structure and properties, especially the water holding capacity of these polysaccharides.

\section{EXPERIMENTAL}

\section{Materials}

Agarose. One kilogram of the seaweed Gelidium amansii, produced in August 1984 in the Izu Suzaki region (Japan), was suspended in 1.5 liter of 3 percent sodium hydroxide aqueous solution. The suspension was stirred at $60^{\circ} \mathrm{C}$ for one hour. After the alkali pretreatment, extraction was carried out by autoclaving at $128,130,132$, and $134^{\circ} \mathrm{C}$ in 1.5 liter of water successively. The agarose was isolated from each extract by the same method described previously., ${ }^{4,8}$ After gelation of each extract, the gel was cut into strings and kept frozen for about $15 \mathrm{~h}$. The frozen preparations were thawed in running water and dried in vacuo at $40^{\circ} \mathrm{C}$.

The dried specimens were swollen in water 
at $40^{\circ} \mathrm{C}$ overnight, preheated at $70-80^{\circ} \mathrm{C}$ for 30 minutes, and then heated at $105^{\circ} \mathrm{C}$ for $10-15$ minutes using an autoclave. The solutions obtained in this way were placed into a mould to give a cylindrical shape for viscoelastic measurements. The gels were kept at $4^{\circ} \mathrm{C}$ for a week to mature and then kept at the temperature of measurement for one hour before measurements.

Kappa-carrageenan. $120 \mathrm{~g}$ of Chondrus crispus produced in August 1984 in Izu Suzaki region (Japan) was washed in water, then dissolved in 21 water at $80^{\circ} \mathrm{C}$ and the solution was filtered. Two liters of $0.125 \mathrm{moll}^{-1}$ aqueous $\mathrm{KCl}$ was gradually added to the solution, which was then stirred vigorously. After the soluble part was washed out, the kappa-carrageenan was precipitated by $\mathrm{KCl}$ and separated by centrifugation. The excess $\mathrm{KCl}$ in the specimen was eliminated by dialysis and the kappa-carrageenan was dried in vacuo. The potassium content in the specimen measured by atomic absorption spectrophotometry was $2.96 \%$.

The sulfate content in agarose and in kappacarrageenan was quite different: the sulfur content determined by elemental analysis was $0.05 \%$ for agarose and $6.4 \%$ for kappacarrageenan.

The observed values of the intrinsic viscosity for the specimens purified in this way, in
$0.01 \mathrm{~mol}^{-1} \mathrm{NaSCN}$ aqueous solution at 35 ${ }^{\circ} \mathrm{C}$, were $5.4,3.9,3.1$, and $2.0\left(100 \mathrm{ml} \mathrm{g}^{-1}\right)$ for four fractions of agarose extracted at $128,130,132$, and $134^{\circ} \mathrm{C}$, and $13.3(100 \mathrm{ml}$ $\mathrm{g}^{-1}$ ) for kappa-carrageenan, respectively. These values are shown with other parameters in Table I.

The alkali metal salts $\mathrm{LiCl}, \mathrm{NaCl}, \mathrm{KCl}$, and $\mathrm{CsCl}$ used in this study were the extra fine grade reagents (Wako Junyaku Kogyo). The test pieces of these carrageenan gels for rheological and thermal measurements were prepared in the same way described previously. ${ }^{4,8}$

\section{Measurement}

Dynamic viscoelasticity was measured at $2.5 \mathrm{~Hz}$ at the temperature range from 5 to $75^{\circ} \mathrm{C}$ by use of a Rheolograph (CV-100 Toyo Seiki Seisakusho Co., Ltd.). A cylindrically moulded gel $(20 \mathrm{~mm}$ diameter and $30 \mathrm{~mm}$ height) was subjected to forced longitudinal vibration.

The endothermic peak temperature and the endothermic enthalpy accompanying a gelsol transition were observed by use of a DSC (SSC 580 Seiko Electronics Ltd.). The gels used were annealed at $5^{\circ} \mathrm{C}$ for two days, and $42 \pm 0.1 \mathrm{mg}$ of which were put into silver pans (70 $\mu \mathrm{l})$ using vinyl tubes. The temperature was raised from $8^{\circ} \mathrm{C}$ at a rate of $2 \mathrm{~K} \mathrm{~min}^{-1}$.

The volume or weight change of gels in-

Table I. Intrinsic viscosity, content of 3,6-anhydro-L-galactose, quantity of sulfate esters and enthalpy, $\Delta H_{\mathrm{m}}$ of formation of network junctions for agarose

and kappa-carrageenan samples

\begin{tabular}{|c|c|c|c|c|c|}
\hline \multirow{2}{*}{ Sample } & \multirow{2}{*}{$\begin{array}{c}\begin{array}{c}\text { Extraction } \\
\text { temperature }\end{array} \\
{ }^{\circ} \mathrm{C}\end{array}$} & \multirow{2}{*}{$\begin{array}{c}\begin{array}{c}\text { Intrinsic } \\
\text { viscosity }\end{array} \\
100 \mathrm{ml} \mathrm{g}^{-1}\end{array}$} & \multirow{2}{*}{$\frac{\begin{array}{c}\text { 3,6-Anhydro- } \\
\text { L-galactose }\end{array}}{\%}$} & \multirow{2}{*}{$\frac{\text { Sulfate ester }}{\mathrm{mol} / \mathrm{C}_{6} \mathrm{H}_{10} \mathrm{O}_{5}}$} & \multirow{2}{*}{$\frac{-\Delta H_{\mathrm{m}}}{\mathrm{kJ} \mathrm{mol}^{-1}}$} \\
\hline & & & & & \\
\hline $\mathrm{F} 1$ & 134 & 2.0 & 44.2 & 0.0021 & $1.3 \times 10^{3}$ \\
\hline F2 & 132 & 3.1 & & 0.0018 & \\
\hline F3 & 130 & 3.9 & & 0.0022 & \\
\hline $\mathrm{F} 4$ & 128 & 5.4 & 43.8 & 0.0024 & $1.3 \times 10^{3}$ \\
\hline Kappa-carrageenan & 80 & 13.3 & & 0.355 & 38 \\
\hline
\end{tabular}

${ }^{\text {a }}$ Intrinsic viscosity was determined in $0.01 \mathrm{moll}^{-1}$ sodium thiocyanate aqueous solution at $35^{\circ} \mathrm{C}$ by using Ubbelohde viscometer. 
duced by immersion in DMSO- $\mathrm{H}_{2} \mathrm{O}$ or in ethanol at $25 \pm 0.1^{\circ} \mathrm{C}$ for $24 \mathrm{~h}$ was observed by the same method described previously. ${ }^{8,9}$ The initial volume for the volume change measurement was $20 \mathrm{ml}$. The initial weight for the weight change measurement was $40 \mathrm{~g}$.

\section{RESULTS AND DISCUSSION}

\section{Temperature Dependence of Elastic Modulus} of Agarose and Kappa-Carrageenan Gels

The temperature dependences of dynamic elastic modulus $E^{\prime}$ of agarose and kappacarrageenan gels of various concentrations are shown in Figures 2 and 3. As is seen from these figures, there is a remarkable difference in the temperature dependence. At first, $E^{\prime}$ of agarose gels increases with increasing temperature in a temperature range from 5 to $35^{\circ} \mathrm{C}$, while $E^{\prime}$ of kappa-carrageenan gels gradually decreases in the same range. Secondly, the temperature $T_{0}$ at which $E^{\prime}$ begins to decrease rapidly is almost independent of concentration for agarose gels, while that for kappa-carrageenan gels is shifted to higher temperature with increasing concentration. The temperature $T_{0}$ may be defined as the temperature at which the slope of a straight line tangent to a smoothed curve connecting observed values changes most remarkably. Agarose molecule contains many hydroxyl groups and then stable microcrystalline regions are formed in agarose gels by hydrogen bondings between molecules. On the other hand, the formation and aggregation of helical parts in kappa-carrageenan molecules are hindered by electrostatic repulsion due to the sulfate groups in molecules.

The temperature dependence of elastic modulus of thermo-reversible gels was recently explained $^{14}$ by a simple model consisting of two regions: the crystalline-like region acting as junction zones and the amorphous region consisting of long flexible chains. A statistical calculation based on this model can explain qualitatively the temperature dependence of
$E^{\prime}$ shown in Figures 2 and 3. According to this theory, the elastic modulus of thermoreversible gels increases with increasing temperature monotonically provided that the bonding energy $\varepsilon$ or the ceiling number $v$ of segments which are liberated from junction zones is sufficiently high. If the number of

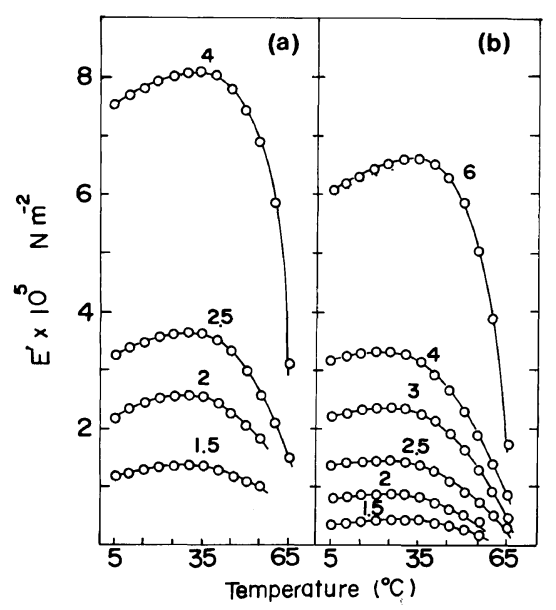

Figure 2. Temperature dependence of dynamic Young's modulus $E^{\prime}$ for agarose gels of various concentrations: (a) $[\eta]=5.4\left(100 \mathrm{ml} \mathrm{g}^{-1}\right)$ at $35^{\circ} \mathrm{C}$ in $0.01 \mathrm{moll}^{-1} \mathrm{NaSCN}$; (b) $[\eta]=2.0\left(100 \mathrm{ml} \mathrm{g}^{-1}\right)$ at $35^{\circ} \mathrm{C}$ in $0.01 \mathrm{moll}^{-1} \mathrm{NaSCN}$. The figures beside the curves represent the concentration in $\%(w / w)$.

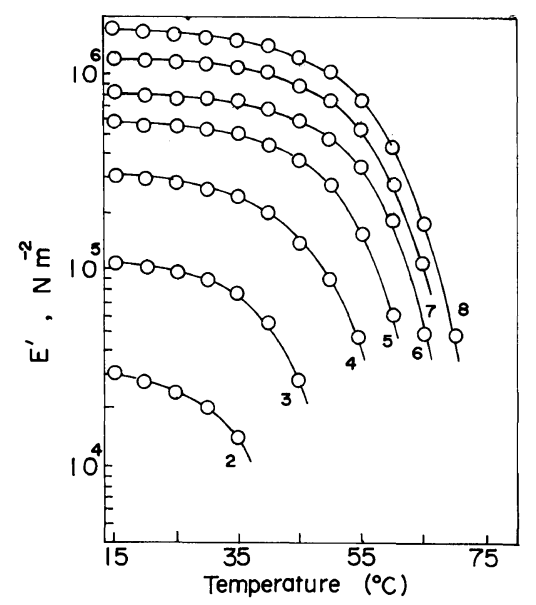

Figure 3. Temperature dependence of dynamic Young's modulus $E^{\prime}$ for kappa-carrageenan gels of various concentrations. The figures beside the curves represent the concentration in $\%(w / w)$. 
segments liberated from junction zones becomes larger than an upper limit, the gel-sol transition occurs. For a lower bonding energy $\varepsilon$ or for a smaller value of $v$, the elastic modulus decreases monotonically with increasing temperature. For intermediate values of $\varepsilon$ and $v$, the elastic modulus increases up to a certain temperature $T_{0}$ and then decreases with increasing temperature. Therefore, the bonding energy $\varepsilon$ or the ceiling number $v$ is larger in agarose than is in kappa-carrageenan. This is reasonable if we assume the first possibility because there are many hydroxyl groups in agarose and many sulfate groups in kappa-carrageenan. As for $v$, it is not possible to evaluate it from the present experimental data alone. As for the difference of $E^{\prime}$ between $\mathrm{F} 1$ and $\mathrm{F} 4$ as shown in Figure $2, E^{\prime}$ is larger in F4 gel than is in F1 gel for the same concentration and temperature. This tendency is the same as observed previously; agarose gels of higher molecular weight has a larger elastic modulus than those of lower molecular weight. ${ }^{13}$

If the theory of rubber elasticity is applicable, ${ }^{15}$ the number average molecular weight $\bar{M}_{c}$ of the chain adjoining adjacent crosslinks can be estimated from $M_{c}=3 \rho R T / E$, where $\rho$ is the density of the gel, $R$ the gas constant per mole, $T$ the absolute temperature and $E$ the Young's modulus. Substitution of $E=$ $8 \times 10^{5} \mathrm{Nm}^{-2}$ for an F1 gel, $E=3.3 \times 10^{5}$ $\mathrm{N} \mathrm{m}^{-2}$ for an F4 gel, and $E=3.0 \times 10^{5} \mathrm{~N} \mathrm{~m}^{-2}$ for a kappa-carrageenan gel each of concentration $4 \%$ at room temperature gives the number of structural units as 31 for the F1 gel, 75 for the F4 gel and 66 for the kappa-carrageenen gel where the molecular weight of structural unit is taken as 306 $\left(=\mathrm{C}_{12} \mathrm{H}_{18} \mathrm{O}_{9}\right)$ for agarose and 385 $\left(=\mathrm{C}_{12} \mathrm{H}_{17} \mathrm{O}_{12} \mathrm{~S}\right)$ for kappa-carrageenan (see Figure 1). The above values of the number of structural units is too small so that the theory of rubber elasticity may be applicable. It is probably due to the fact that molecular network chains in polysaccharide gels are very stiff as suggested by several workers. ${ }^{16,17}$ This is also the reason why we have used a Langevin chain rather than a Gaussian chain in order to explain the temperature dependence of the elasticity of thermo-reversible gels ${ }^{14}$ as stated above briefly.

\section{DSC Curves of Agarose and Kappa-Carra- geenan Gels}

Heating DSC curves of agarose and kappa-carrageenan gels of various concentrations are shown in Figures 4-6. The broken lines are base lines used for estimating endothermic enthalpy from the area surrounded by the DSC curve and the base line. The endothermic peaks accompanying gelsol transitions appear at about $70^{\circ} \mathrm{C}$ and 85 ${ }^{\circ} \mathrm{C}$ for the fraction of agarose gels with $[\eta]=$ 2.0 (Figure 4) and 5.4 (Figure 5). Each temperature at peak is almost independent of concentration. The peak is shifted to higher temperatures with increasing molecular weight of the fraction. This tendency corresponds

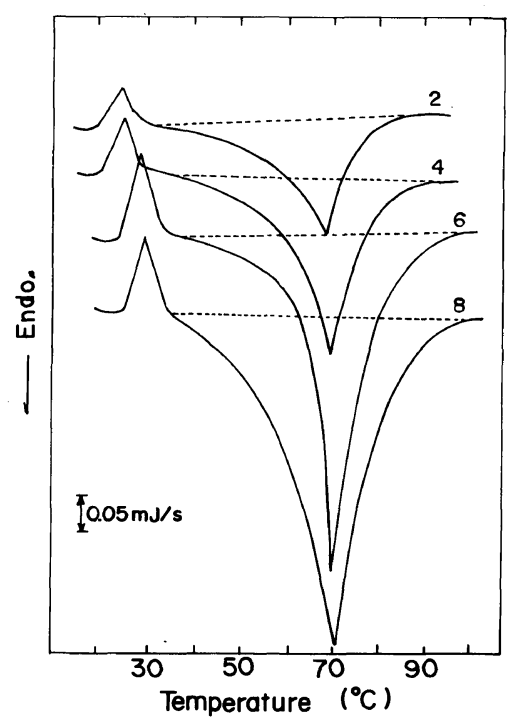

Figure 4. Heating DSC curves for agarose gels of various concentrations. $[\eta]=2.0\left(100 \mathrm{ml} \mathrm{g}^{-1}\right)$ at $35^{\circ} \mathrm{C}$ in $0.01 \mathrm{moll}^{-1} \mathrm{NaSCN}$. The figures beside the curves represent the concentration in $\%(w / w)$. The broken line represents the base line for the endothermic peak. 


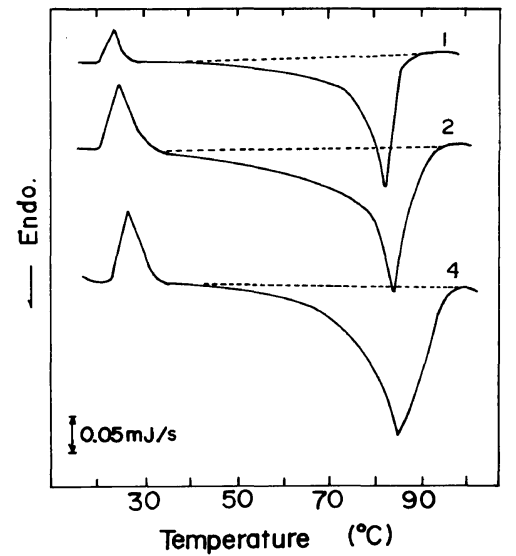

Figure 5. Heating DSC curves for agarose gels of various concentrations. $[\eta]=5.4\left(100 \mathrm{ml} \mathrm{g}^{-1}\right)$ at $35^{\circ} \mathrm{C}$ in $0.01 \mathrm{moll}^{-1} \mathrm{NaSCN}$. The figures beside the curves represent the concentration in $\%(w / w)$. The broken line represents the base line for the endothermic peak.

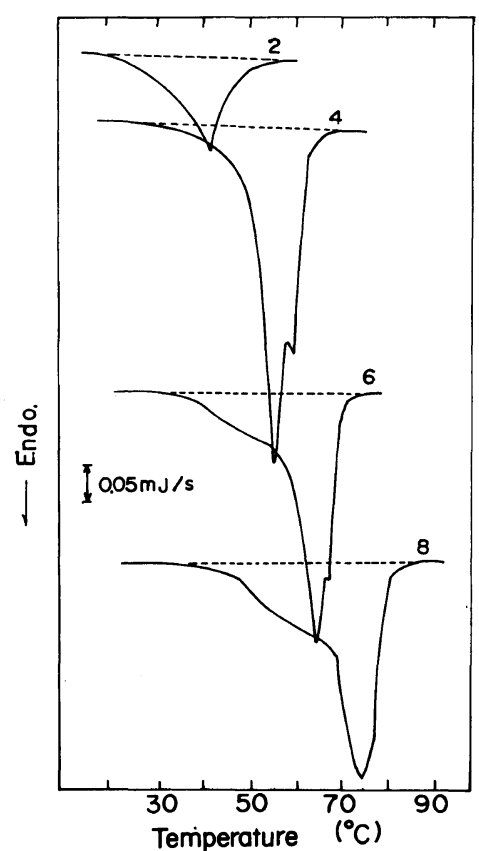

Figure 6. Heating DSC curves for kappa-carrageenan gels of various concentrations. The figures beside the curves represent the concentration in $\%(w / w)$. The broken line represents a base line for the endothermic peak.

well to the results for $E^{\prime}$ observed in the previous section.

An exothermic peak appears at about 30

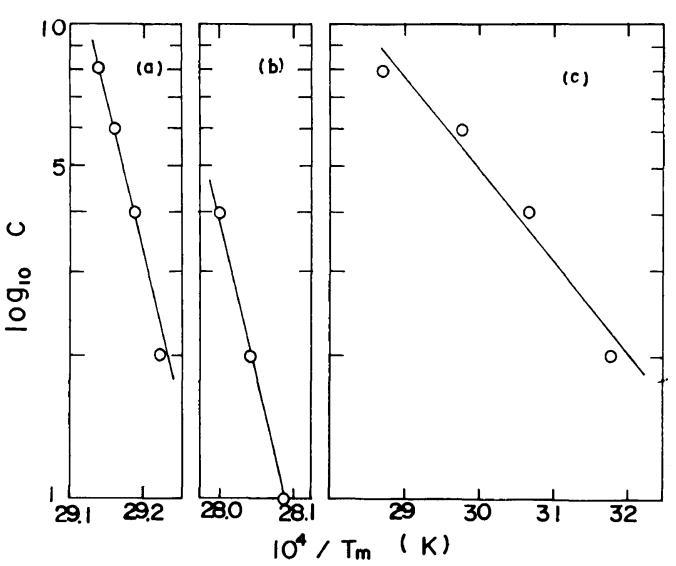

Figure 7. Eldridge-Ferry plot for agarose and kappacarrageenan gels. $c$, concentration of gels; $T_{\mathrm{m}}$, melting point; (a), agarose with $[\eta]=2.0\left(100 \mathrm{ml} \mathrm{g}^{-1}\right)$; (b), agarose with $[\eta]=5.4\left(100 \mathrm{mlg}^{-1}\right) ;(\mathrm{c})$, kappa-carrageenan:

${ }^{\circ} \mathrm{C}$ for agarose gels, and is shifted to higher temperatures with increasing concentrations for both fractions with $[\eta]=2.0$ and $[\eta]=5.4$ (see Figures 4 and 5). This exothermic peak shifted to higher temperatures after the temperature was once raised up to $35^{\circ} \mathrm{C}$ and then lowered to $5^{\circ} \mathrm{C}$ although the data are not shown here.

By the repetition of heating and cooling, the exothermic peak was shifted to higher temperatures. The syneresis is promoted by this operation, and at the same time, the molecules might be rearranged to favour the crystallization. This phenomenon is especially observed in agarose or agar molecules in which there are many hydroxyl groups. ${ }^{18,19}$ In kappa-carrageenan molecules, this exothermic peak was not observed. The exothermic peak temperature is slightly lower than the temperature at which $E^{\prime}$ showed maximum in Figures 2 and 3.

Figure 7 shows the Eldridge-Ferry plot ${ }^{20}$ for agarose and kappa-carrageenan gels:

$$
\log _{10} c=\frac{\Delta H_{m}}{2.303 R T_{m}}+\text { const. }
$$

Here, $-\Delta H_{m}$ represents the heat absorbed on forming a mole of the junction points 
that stabilize the network structure of the gel, and $c$ and $T_{m}$ stand for concentration and melting temperature of gels respectively.

The plots show some curvature concave to the $1 / T_{m}$ axis but mean values of $\Delta H_{m}$ can be obtained from the best straight line drawn through the points. The values of $-\Delta H_{m}$ are $38 \mathrm{~kJ} \mathrm{~mol}^{-1}$ for kappa-carrageenan gels and $1.3 \times 10^{3} \mathrm{~kJ} \mathrm{~mol}^{-1}$ for agarose gels of both fractions. The fact that $-\Delta H_{m}$ is much larger in agarose than in kappa-carrageenan corresponds well to the comment mentioned at the last part of the previous section.

Volume or Weight Change of Agarose and Kappa-Carrageenan Gel by Immersion in DMSO-Water or in Ethanol

The observation of volume or weight change in gels by immersion in an alkali metal salt solution or in an organic solvent gives a clue to understanding structure and properties of the gels. The volume changes of agarose and kappa-carrageenan gels by immersion in the mixture DMSO-water for $24 \mathrm{~h}$ are shown in Figures 8 and 9. The volume of agarose gels decreases only slightly with increasing mole fraction (mf) of DMSO in four fractions with

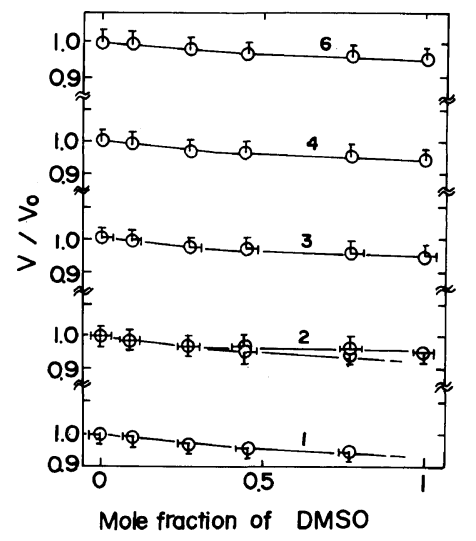

Figure 8. Volume change of agarose gels of various concentrations induced by immersion in DMSO $\cdot \mathrm{H}_{2} \mathrm{O}$ vs. mole fraction of DMSO. $\bullet,[\eta]=5.4 ; Q,[\eta]=3.9 ;$ G, $[\eta]=3.1 ; \delta,[\eta]=2.0$. The figures beside the curves represent the initial concentration of gels in $\%(w / w)$. $[\eta]=2.0,[\eta]=3.1,[\eta]=3.9$, and $[\eta]=5.4$ for the concentrations of gels from 1 to $6 \%(\mathrm{w} / \mathrm{w})$ (Figure 8).

The volume of kappa-carrageenan gels is almost constant at the concentration of DMSO from 0 to $0.33 \mathrm{mf}$, and it increases drastically beyond that DMSO mole fraction

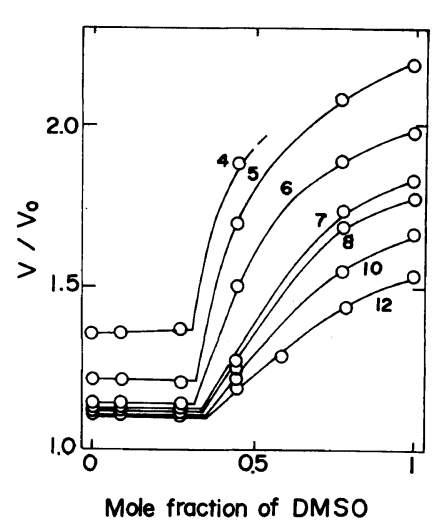

Figure 9. Volume change of kappa-carrageenan gels of various concentrations induced by immersion in DMSO $\cdot \mathrm{H}_{2} \mathrm{O} v s$. mole fraction of DMSO. The figures beside the curves represent the initial concentration of gels in $\%(w / w)$.

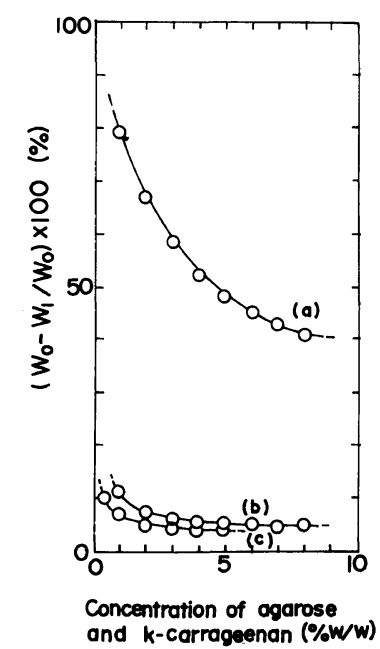

Figure 10. Weight change of kappa-carrageenan (a) and agarose gels: $(\mathrm{b}),[\eta]=2.0\left(100 \mathrm{ml} \mathrm{g}^{-1}\right) ;(\mathrm{c}),[\eta]=$ $5.4\left(100 \mathrm{ml} \mathrm{g}^{-1}\right) v s$. the initial concentration of gels in $\%$ (w/w). Weight change induced by immersion in ethanol. $W_{0}$, weight before immersion, $W_{1}$, weight after immersion. 
(Figure 9). This increase of gel volume is more remarkable in dilute gels than in concentrated gels. A $4 \%(w / w)$ kappa-carrageenan gel swells very rapidly, and it is dissolved at high DMSO concentration.

DMSO is a powerful polar solvent for polysaccharides. Thermodynamical measurements, ${ }^{21}$ NMR investigation, ${ }^{22}$ and ultrasonic study ${ }^{23}$ revealed that DMSO interacts with water and forms $\left(\mathrm{CH}_{3}\right)_{2} \mathrm{SO} \cdot 2 \mathrm{H}_{2} \mathrm{O}$ or $\left(\mathrm{CH}_{3}\right)_{2} \mathrm{SO} \cdot 3 \mathrm{H}_{2} \mathrm{O}$. The mole fraction 0.33 corresponds to a molecular ratio of the dihydrate compounds of DMSO. The solubility of polysaccharides will increase beyond the DMSO mole fraction 0.33 with increasing DMSO mole fraction, because the quantity of non-hydrated DMSO increases.

The weight change of agarose and kappacarrageenan gels induced by immersion in ethanol is shown in Figure 10. The weight change of agarose gels is very small, while that of carrageenan attained from 40 to $80 \%$. It is noteworthy that the weight change is more remarkable in dilute gels than in concentrated gels as in the case of volume change (Figure 9).

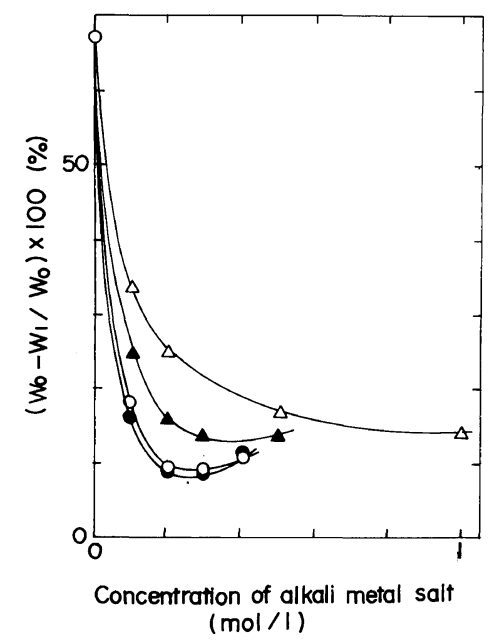

Figure 11. Weight change of kappa-carrageenan gels (initial concentration: $2 \%(\mathrm{w} / \mathrm{w})$ ) containing alkali metal salt $v s$. concentration of alkali metal salt. Weight change induced by immersion in ethanol. $W_{0}$, weight before immersion; $W_{1}$, weight after immersion. $\triangle, \mathrm{LiCl}$; $\mathrm{NaCl}, \mathrm{O}, \mathrm{KCl} ; \mathrm{O}, \mathrm{CsCl}$.
Figure 11 shows the weight change of $2 \%$ $(\mathrm{w} / \mathrm{w})$ kappa-carrageenan gels, containing alkali metal salt of various concentrations, by immersion in ethanol at $25^{\circ} \mathrm{C}$ for $24 \mathrm{~h}$. The kappa-carrageenan gel containing $\mathrm{CsCl}$ (O) or $\mathrm{KCl}(\bigcirc)$ showed the smaller weight change than the gel containing $\mathrm{LiCl}(\triangle)$ or $\mathrm{NaCl}(\boldsymbol{\Delta})$. As is stated in the introductory part, $\mathrm{K}^{+}$or $\mathrm{Cs}^{+}$shields the electrostatic repulsion of sulfate groups in kappacarrageenan molecules more directly than $\mathrm{Li}^{+}$or $\mathrm{Na}^{+}$. Thus, the helical molecules are more densely packed in the gel containing $\mathrm{K}^{+}$ or $\mathrm{Cs}^{+}$than in the gel containing $\mathrm{Li}^{+}$or $\mathrm{Na}^{+}$. Therefore, the quantity of water exuded out from the gel is less in the gel containing $\mathrm{K}^{+}$or $\mathrm{Cs}^{+}$than in the gel containing $\mathrm{Li}^{+}$or $\mathrm{Na}^{+}$. This tendency is consistent with the results observed previously ${ }^{4}$; kappa-carrageenan gels containing $\mathrm{K}^{+}$or $\mathrm{Cs}^{+}$showed a maximum of $E^{\prime}$ as a function of the concentration of $\mathrm{K}^{+}$or $\mathrm{Cs}^{+}$at about $0.2 \sim 0.3 \mathrm{moll}^{-1}$. The gels containing $\mathrm{K}^{+}$or $\mathrm{Cs}^{+}$take the most densely packed structure at this concentration of ions. Therefore, the quantity of water exuded out from the gels is the smallest at this concentration of $\mathrm{K}^{+}$or $\mathrm{Cs}^{+}$. Thus, the water holding capacity of kappa-carrageenan gels has a close relation with the elastic modulus and

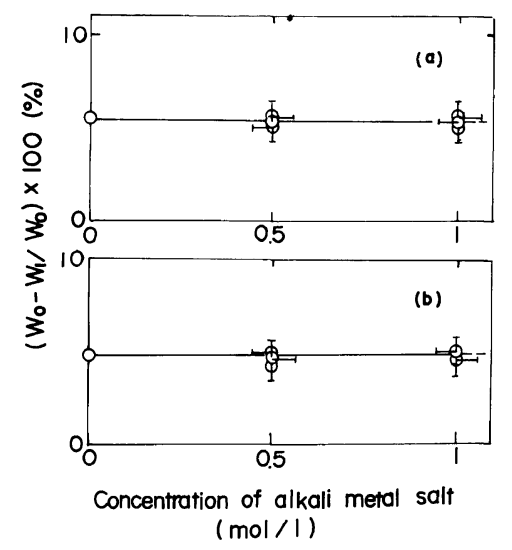

Figure 12. Weight change of $2 \%(w / w)$ (a) and $4 \%$ $(\mathrm{w} / \mathrm{w})$ (initial concentration) (b) agarose gels $v s$. concentration of alkali metal salt. $\Phi, \mathrm{LiCl} ; \circlearrowleft, \mathrm{NaCl} ; \Phi$, $\mathrm{KCl} ; \bullet, \mathrm{CsCl}$. 
the weight change induced by immersion in ethanol. The 4\% kappa-carrageenan gels containing alkali metal salt showed a similar tendency although the observed data are not shown here. In this case, the weight change of the gels is less remarkable than in $2 \%$ gels.

The weight change of $2 \%$ and $4 \%(w / w)$ agarose gels, containing alkali metal salt of various concentrations, induced by immersion in ethanol at $25^{\circ} \mathrm{C}$ for $24 \mathrm{~h}$ is shown in Figure 12. As is seen from this figure, the weight change is almost negligible. These findings are consistent with the earlier observation..$^{4,8}$

In conclusion, the difference of rheological and thermal behaviour between agarose and kappa-carrageenan gels is quite certainly confirmed irrespective of the origin of polysaccharides. Agarose gels show larger elastic modulus and higher melting temperature than kappa-carrageenan gels. The heat absorbed on forming a mole of the junction points is far larger in agarose gels than in kappa-carrageenan gels according to the analysis suggested by Eldridge and Ferry. The water holding capacity is much higher in agarose gels than in kappa-carrageenan gels judging from the volume or weight change induced by immersion in organic solvents.

\section{REFERENCES}

1. D. A. Rees, Pure Appl. Chem., 53, 1 (1981).

2. D. A. Rees, E. R. Morris, D. Thom, and J. K. Madden, "The Polysaccharides," Vol. 1, G. O. Aspinall, Ed., Academic Press, New York, N. Y., 1982, pp 195-290.

3. C. F. Lin and P. M. T. Hansen, Macromolecules, 3, 269 (1970).

4. M. Watase and K. Nishinari, Rheol. Acta, 21, 318
(1982).

5. M. Watase and K. Nishinari, Makromol. Chem., in press.

6. M. Watase and K. Nishinari, Makromol. Chem., in press.

7. M. Watase and K. Nishinari, "Gums and Stabilisers for the Food Industry," Vol. 3, G. O. Phillips, D. Wedlock, and P. Williams, Ed., Elsevier, Amsterdam, 1986, p 185.

8. M. Watase and K. Nishinari, Colloid Polym. Sci., 260, 271 (1982).

9. M. Watase and K. Nishinari, "Gums and Stabilisers for the Food Industry," Vol. 2, G. O. Phillips, D. Wedlock, and P. Williams, Ed., Pergamon Press, Oxford, 1984, p 541.

10. J. F. Millero, "Water and Aqueous Solutions," R. A. Horne, Ed., Wiley-Interscience, New York, N. Y., 1972 , p 519.

11. M. Watase and K. Nishinari, Nippon Shokuhin Kogyo Gakkaishi, 28, 437 (1981).

12. K. Nishinari and M. Watase, Carbohydr. Polym., 3, 39 (1983).

13. M. Watase and K. Nishinari, Rheol. Acta, 22, 580 (1983).

14. K. Nishinari, S. Koide, and K. Ogino, J. Phys. (Paris), 46, 793 (1985).

15. L. R. G. Treloar, "The Physics of Rubber Elasticity," 3rd ed, Clarendon Press, Oxford, 1975, p 65.

16. O. Smidsrod, A. Haug, and B. Lian, Acta Chem. Scand., 26, 71 (1972).

17. E. Bailey, J. R. Mitchell, and J. M. V. Blanshard, Colloid Polym. Sci., 255, 856 (1977).

18. M. Watase and K. Nishinari, in the same literature cited as ref 7, 1986, p 535.

19. M. Watase and K. Nishinari, Colloid Polym. Sci., 264, 877 (1986).

20. J. E. Eldridge and J. D. Ferry, J. Phys. Chem., 58, 992 (1954).

21. S. A. Schichman and R. L. Amey, J. Phys. Chem., 75, 98 (1971).

22. D. E. Bowen, M. A. Priesand, and M. P. Eastman, $J$. Phys. Chem., 78, 2611 (1974).

23. K. J. Packer and D. J. Tomlinson, Trans. Faraday Soc., 67, 1302 (1971). 